\title{
A qualitative classification of extraterrestrial civilizations
}

\author{
Valentin D. Ivanov ${ }^{1}$, Juan Carlos Beamín² ${ }^{2}$ Claudio Cáceres ${ }^{3,4}$, and Dante Minniti ${ }^{3,5}$ \\ ${ }^{1}$ European Southern Observatory, Karl-Schwarzschild-Str. 2, 85748 Garching bei München, Germany \\ e-mail: vivanov@eso.org \\ ${ }^{2}$ Núcleo de Astroquímica y Astrofísica, Instituto de Ciencias Químicas Aplicadas, Facultad de Ingeniería, Universidad Autónoma de \\ Chile, Av. Pedro de Valdívia 425, Santiago, Chile \\ ${ }^{3}$ Departamento de Ciencias Físicas, Facultad de Ciencias Exactas, Universidad Andrés Bello, Av. Fernandez Concha 700, \\ Las Condes, Santiago, Chile \\ ${ }^{4}$ Núcleo Milenio Formación Planetaria - NPF, Universidad de Valparaíso, Av. Gran Bretaña 1111, Playa Ancha, Casilla 5030, \\ Valparaíso, Chile \\ ${ }^{5}$ Vatican Observatory, V00120 Vatican City State, Italy
}

Received 28 January 2020 / Accepted 26 May 2020

\begin{abstract}
Context. Interest in searches for extraterrestrial civilizations (ETCs) has been boosted in recent decades by the discovery of thousands of exoplanets.

Aims. We turn to the classification of ETCs for new considerations that may help to design better strategies for searching for ETCs. Methods. This study is based on analogies with our own biological, historical, technological, and scientific development. We took a basic taxonomic approach to ETCs and investigated the implications of the new classification on ETC evolution and observational patterns. Finally, we used the quantitative scheme of Kardashev and considered its implications on the searches for ETCs as a counter example to our qualitative classification.

Results. We propose a classification based on the abilities of ETCs to modify and integrate with their environments: Class 0 uses the environment as it is, Class 1 modifies the environment to fit its needs, Class 2 modifies itself to fit the environment, and a Class 3 ETC is fully integrated with the environment. Combined with the classical Kardashev scale, our scheme forms a two-dimensional method for interpreting ETC properties.

Conclusions. The new framework makes it obvious that the available energy is not a unique measure of ETC progress: it may not even correlate with how well that energy is used. The possibility for progress without increased energy consumption implies a lower detectability, so in principle the existence of a Kardashev Type III ETC in the Milky Way cannot be ruled out. This reasoning weakens the Fermi paradox, allowing for the existence of advanced, yet not energy hungry, low-detectability ETCs. The integration of ETCs with the environment will make it impossible to tell technosignatures and natural phenomena apart. Therefore, the most likely opportunity for SETI searches to find advanced ETCs is to look for beacons, specifically set up by them for young civilizations like ours (if they would want to do that remains a matter of speculation). The other SETI window of opportunity is to search for ETCs at technological level similar to ours. To rephrase the famous saying of Arthur Clarke, sufficiently advanced civilizations are indistinguishable from nature.
\end{abstract}

Key words. extraterrestrial intelligence - astrobiology - history and philosophy of astronomy

\section{Introduction}

On a fundamental level, the search for extraterrestrial civilizations (ETCs) is motivated by scientific curiosity. We want to understand how intelligent life and intelligence arise and evolve, to compare biologies, histories, and social structures that have developed completely independently from each other. Undoubtedly, contact with an ETC carries potential risks (Neal 2014), but there are also indications that it may have a stimulating effect on humanity (Kwon \& Bercovici 2017). In the long run, the transfer of new knowledge from fundamental sciences to industry is likely to induce fast economic growth, and on a purely psychological level we will have for the first time a truly external - with respect to the human kind - scale to measure up ourselves and our achievements.

However, all these promising prospects ultimately require a successful search for extraterrestrial intelligence (SETI). The SETI programs in the last half century have been fruitless. One possibility is that the Universe is empty, but the commonly found ingredients of life (at least as we know it) cast some doubts on this hypothesis. Another option is that the ETCs are extremely rare. Therefore, success is just a matter of time and increased sensitivity of the techniques we are already applying. Without listing all the possibilities for the silentium universi, let us consider the possibility that our search strategies may be wrong. Indeed, some time ago Bradbury et al. (2011) pointed to certain caveats in our strategies: SETI mostly remains an effort isolated from the wider astronomical and astrobiological studies, and the SETI proponents refuse to adopt a broader multidisciplinary approach and to consider important criticism.

So far, the dominant SETI approach, going back to Cocconi \& Morrison (1959), relies on (i) the willingness of ETCs to be detected or (ii) their unwillingness or failure to control their own energy waste (that we could detect). In the former case we are searching for radio beacons set up with the purpose of being visible to other ETCs, and in the latter for the emission that would leak into space in the course of regular radio communications. The transfer of the searches to other ranges 
of the electromagnetic spectrum does not make a fundamental difference (Fabian 1977; Wright et al. 2014a).

Kardashev (1964) evaluated the feasibility of these approaches in radio and as a by-product developed a quantitative scheme to measure the stage of an ETC's advancement via the total amount of energy they have at their disposal. Undoubtedly, Kardashev's classification is still useful for SETI because it helps to define benchmark energy capabilities of the ETCs and thus to set up sensitivity requirements for the SETI equipment. However, some new considerations have arisen in recent years. For example, Benford et al. (2010a,b) argued about costoptimized means of interstellar communication, and proposed some strategies to that effect (see more arguments for cost-saving in Davies \& Wagner 2013). Kardashev (1964) had not taken these into account. He implicitly makes the assumption that unlimited resources are available to the ETCs, allowing unconstrained growth of the energy production and consumption, at least up to a galactic scale. Furthermore, the estimates of Kardashev are upper limits that give the maximum energy available for interstellar communication for the given level of ETC development.

Our historic and modern experience can hardly support unconstrained growth. Therefore, it is compelling to re-examine the ETC classification as a guiding tool for SETI strategies, aiming to optimize them and to reach a new priority scale for the different search methods. The next section reviews Kardashev's classification and its implications. Sections 3, 4, and 5 describe a new quantitative scale proposed here and its implications. Section 6 summarizes this work.

\section{Quantitative classification: Kardashev's scale}

Kardashev (1964) introduced a classification scheme for ETCs based on the energy available to them. This is a quantitative approach, well justified in the context of that study, aimed to determine the technical feasibility of the communication between civilizations. He considered an isotropic radio emission and estimated that sending information with a data rate of $3 \times 10^{5}$ bits s$^{-1}$ at distance of $\sim 3 \mathrm{Mpc}$ required transmitting power of $4 \times 10^{33} \mathrm{erg} \mathrm{s}^{-1}$, comparable to the total solar luminosity. The adopted limit of $\sim 3 \mathrm{Mpc}$ is just below the distances to the M81 and Cen A groups of galaxies and encompasses a large number of galaxies, significantly increasing the number of ETCs that can potentially be detected, in contrast with more modest emitting power that would limit an ETC search to nearby stars.

Kardashev (1964) concluded that the transmitting power is the controlling parameter of the data rate and covered distance. This prompted him to build a classification of ETCs based on the energy in their disposal:

- Class I: an ETC in possession of all energy of its planet or $\sim 4 \times 10^{19} \mathrm{erg} \mathrm{s}^{-1}$;

- Class II: an ETC in possession of all energy of its star or $\sim 4 \times 10^{33}$ erg s$^{-1}$;

- Class III: an ETC in possession of all energy of its galaxy or $\sim 4 \times 10^{44} \mathrm{erg} \mathrm{s}^{-1}$.

The first Class is the easiest to comprehend, because it implies a technological level close to that of present-day Earth. The humanity itself is approaching this level of energy consumption. Right now, we are still mostly limited to the Earth's fossil fuel and atomic energy from some radioactive elements; the renewable sources of energy are still underutilized, but their contribution in the total energy budget of our civilization is increasing.
The second Class is more hypothetical. Kardashev (1964) gives the Dyson sphere as an example (Dyson 1960). Such a structure is unstable against collapse, as pointed out by many authors (e.g., Suffern 1977). This problem can be addressed without calling for speculative technology or physics by breaking the sphere into a swarm of individual elements, often called a Dyson swarm. Each of these elements is not unlike the space habitats proposed by O'Neill \& Kraus (1979), but they must be quite numerous to provide a covering factor close to unity, so nearly the entire energy of the star is captured - as required by the definition of the Class II ETC.

Dyson (1960), Kardashev (1964) and Sagan \& Walker (1966) realized that the most prominent signature of both the ETCs' energy metabolism and the Dyson sphere would be the infrared (IR) radiation, and a number of searches for stars with IR excesses have been carried out since, mainly at stars on the main sequence that are long lived and are not expected to show IR excess, all with negative results (Slysh 1985; Tilgner \& Heinrichsen 1998; Timofeev et al. 2000; Jugaku \& Nishimura 2004; Carrigan 2009; Wright et al. 2014a; Griffith et al. 2015; Osmanov 2016). Searches in the optical were also considered by Osmanov \& Berezhiani (2018), who predicted anomalous variability of the sphere's structure due to oscillations. Zackrisson et al. (2018) argued that a Dyson sphere with a covering factors less than unity can be recognized as a subluminous source, as long as an accurate parallax measurement is available. They searched for such objects combining the Gaia Data Release 1 (Gaia Collaboration 2016) with the Radial Velocity Experiment (RAVE; Kunder et al. 2017) and found a few stars with lower intrinsic luminosity than expected for their spectral Class, and with no detectable IR excess. However, alternative explanations such as unseen companions that might compromise the astrometric solutions or gray dust cannot be fully excluded.

The last Class in Kardashev's classification is the most speculative, and our current technology gives little clues as to how an ETC could capture and utilize the energy of an entire galaxy. One option is a simple quantitative expansion of Class II ETCs, populating a galaxy with multiple Dyson spheres, whose total number is comparable to this of the stars in the galaxy. Similarly to the sphere-enshrouded stars, the galaxy will become fainter and redder and move away from the usual scaling relations such as the Tully-Fisher relation. Following this argument, Zackrisson et al. (2015) set an upper limit of $\leq 0.3 \%$ to the local Kardashev's Class III disk galaxies.

Wright et al. (2014b,a, 2016) and Griffith et al. (2015) searched for ETCs with large energy supplies, mostly by means of the Wide Field Infrared Survey Explorer (WISE; Wright et al. 2010). They identified some unusual objects, and none of them fully match the expected signatures of Class III ETCs. The authors converted the obtained observational limits into limits on the ETCs' energy supply. Other teams also failed to detect Class III ETCs (Annis 1999; Garrett 2015; Olson 2017).

A possible explanation for the lack of detections is suggested by Lacki (2019), who investigated the observational consequences if only a fraction of the stars is enshrouded. Presumably, it is easier to build Dyson spheres around low-mass stars than around hot high-mass stars for which the habitability zone is further out. The model predicts no detectable effects if the limit is close to the Solar Class stars; it must be raised up to $\sim 30 L_{\text {Sun }}$ to make the presence of Dyson spheres apparent.

Lacki (2016) considered an alternative to the classical Dyson spheres - enshrouding the entire galaxies with artificial dust that would effectively turn them into black boxes, bright only in the microwave spectral region. He searched the Planck Catalog of 
Compact Sources (Planck Collaboration XIII 2016), obtaining a negative result.

Although the last two Classes are purely hypothetical, Kardashev's classification gained foothold in the ETC studies because of its convenience and the straightforward quantitative parameterisation. In his excellent review, Ćirković (2015) showed, with multiple examples, that the scale had had a strong effect on the many SETI searches over the last five decades, on the strategies that these projects have adopted, and on the interpretation of their results.

\section{Qualitative classification of ETCs}

The main motivation to re-examine the existing ETC classification is the question of how a hypothetical ETC would use the available energy beyond the somewhat brute force approach of emitting it in space or blowing things up and building artificial space habitats. In practical terms, we propose measuring this quality of use as the level of interaction with the Universe.

We can turn to humanity's own scientific and technological progress, to trace the capabilities to manipulate matter: mechanical, chemical, atomic, nuclear, etc. One can only speculate on what the next levels will be. Adams \& Laughlin (1997) mentioned the annihilation of CDM particles as a possibility. This is similar to the ETC classification scheme of Barrow (1999), who used the level of manipulation of the microworld as metrics. However, we can generalize further by combining these interactions into a single process: that of modifying our environment. Humanity entered this stage the moment the first tool was used.

From this prospective the next step will be to start modifying ourselves, to match the environment. Modern medicine is on the verge of this transition from curing organisms to upgrading them. It is one step from the gene therapies that prevent a fetus from developing certain dangerous diseases to fetus "improvement". Indeed, the CRISPR-Cas9 (clustered, regularly interspaced, short palindromic repeats) technique for gene editing has recently been improved to allow simultaneous editing of multiple genes (Jinek et al. 2012; Strecker et al. 2019; Camps et al. 2019), bringing both medical and commercial applications of gene therapies within closer reach.

Is modifying ourselves an improvement with respect to modifying the environment? Yes, for a number of reasons. First, because we, as a product of semi-random evolution, are far from optimal for all the environments, even here on the Earth. We have evolved for a short life in small groups, in the savannah. As a result, our brains have insufficient computing capabilities for modern life when we have to complete complex tasks that require us to function within large diverse groups. One of the unfortunate consequences is that we resort to typecasting - a major reason for the problems we face with various biases in the connected global village of today (Kahneman 2011, and the references therein). Vast areas of the Earth's surface near the poles and the oceans are marginally accessible to us.

Next, our bodies wear out quickly, and by the age of 50-60 we start facing problems with such basic components as bones, vision, and hearing. As of 2011, about $0.2-0.3 \%$ of people need hip joint replacement, and $0.1-0.2 \%$ will require knee replacement at some point in their lives (Wengler et al. 2014), and those numbers are increasing (Kremers et al. 2015). In some countries, eight in ten people wear glasses by the age of 20 , and the fraction has long been rising, correlating with reading and education (Williams et al. 2015; Morgana et al. 2018). These are just a few simple problems, we are not discussing the most serious ones such as cancer and various genetic disorders.
A major argument for improving ourselves is to boost our adaptability: an important advantage in a world of nearly infinite environmental variety. We cannot tolerate the entire range of temperatures and pressures without taking major protective measures on our home planet, let alone live on any of the other planets in the Solar System, or, potentially, on any exoplanet.

Last but not least, adopting the strategy to modify ourselves removes the need to achieve consensus about how the environment could be changed, and our civilization has a remarkably poor record regarding agreement, as the two world wars in the last century demonstrated.

We can bracket these two stages of ETC evolution. At the low end of own scale, we extend the term "civilization" to include wild animals that generally use the environment as it is. However, this is not always the case. First, in a broad sense, any given animal modifies the environment, for example simply via its metabolism. Second, there are also well-known examples of animal tool use (Goodall 1964; Van Lawick-Goodall 1971; McGrew 1974, among others), which underlines the point that the new classes that we are about to introduce are not discrete bins, but they represent a part of a continuous sequence.

At the high-end side of our proposed classification, we speculate that the boundary between the environment and oneself would eventually be diluted in the process of self-improvement to the point of merging the two. This is a natural consequence if we assume that the ultimate goal of intelligence is to spread, which in more speculative terms may imply converting all the matter in the Universe into thinking matter (however see Sandberg et al. 2017, for a reasoning why an advanced civilization may prefer to stay dormant during the present cosmological era).

To underline our idea, we use the level of interactions with matter and the degree of integration of ourselves with the environment as near synonymous, because the latter follows on from the former. Historically, once our technological capabilities allowed it, we tried to modify our environment: for example, by moving from natural caves to purpose-built housing. We are already willing to accept the modification of our species, as long as it is seen as upgrading, and even if it is ethically questionable: the tendency toward selective abortions of female fetuses in East Asia proves this (Hesketh et al. 2011).

In summary, we propose a new ETC classification scheme, consisting of the following four categories;

- Class 0: the environment is used as is (animals);

- Class 1: we modify the environment (clothes, buildings);

- Class 2: we modify ourselves to fit the environment (genetically improved humans);

- Class 3: we merge with the environment, converting the dead matter in the Universe into thinking matter.

Throughout this paper, we denote the new classes with Arabic numerals, including fractional classes such as 0.5, 1.3, 2.8 , etc. For clear separation of the Kardashev classes, we use Roman numerals, although there are strong arguments for fractional Kardashev classes as well (see the discussion in Ćirković 2015).

The proposed new ETC scale is less strict than the classical Kardashev scale, as the example given above of tool use by animals suggests. Furthermore, some of the modifications to the environment that we apply right now can also be interpreted as modifications of ourselves. Although, this example is far from the genetic manipulations mentioned earlier; a hand watch and a pair of binoculars are modifications of the environment, but they can also be thought of as removable implants aimed at improving the internal time keeping and the eyesight of average humans. However, eye glasses or lenses, despite being removable 

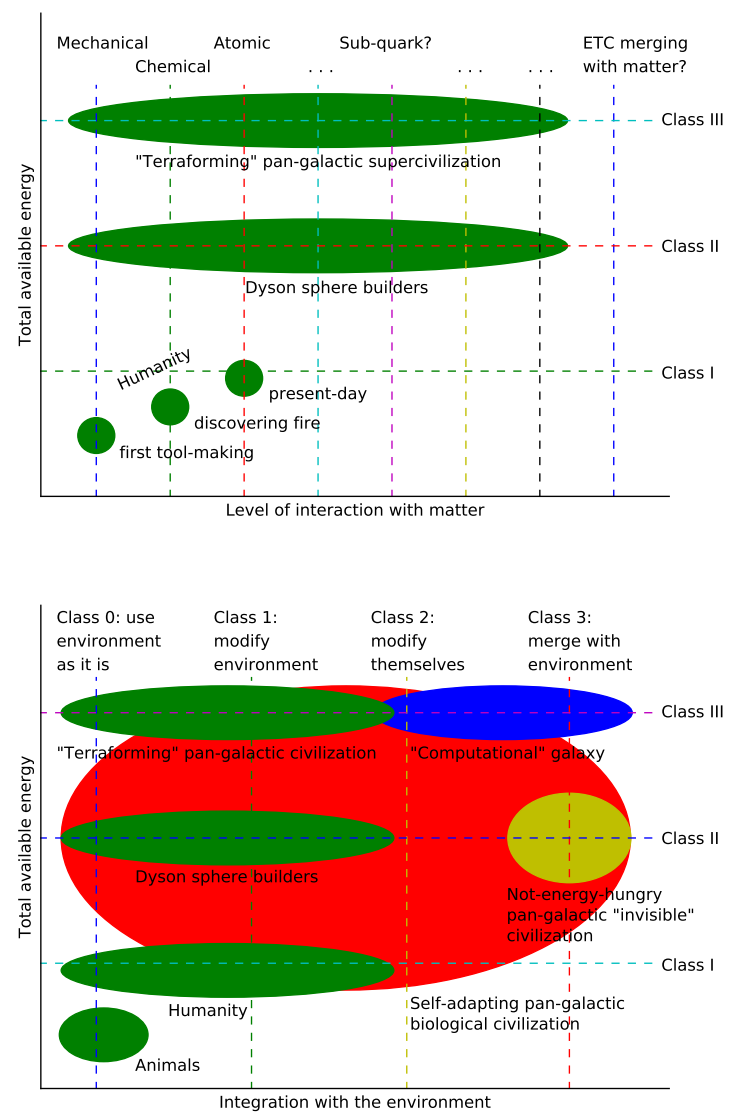

Fig. 1. Two-dimensional classification of the ETCs. Top: horizontal axis expresses the capabilities of an ETC to interact with its environment. Vertical axis quantifies the amount of energy available to them, as defined by the classical Kardashev scale. Bottom: generalized two-dimensional classification. Horizontal axis shows the level of integration with the environment. The approximate locations of humanity throughout history and of a few hypothetical civilizations are shown and labeled. For details, see Sect. 3.

implants, aim to cure a disease, not to advance our capabilities, and therefore are not indications of a self-upgrade.

Some notions of the ideas proposed here can be found in the works of Kecskes $(1998,2013)$, who considered a classification scheme adding the complexity of ETCs' transport, communication, and other resources to the energy resources. In another relevant work, Nunn et al. (2014) considered the historic path of humanity and concluded that it may have reached the point of creating an environmental utopia that removes the stimuli for optimal health, but stops short of asking the question: what is the next step after that utopia? This is a question that we address with our ETC classification scheme.

The two classifications - Kardashev's and the new one we propose - can be combined to form a single two-dimensional scheme that describes the ETC's progress with two parameters: the quantity of the available energy and the quality of its use. Figure 1 demonstrates this scheme with a few examples. The approximate locations of the humanity throughout history are shown on the upper panel: the first tool-making illustrates the mastering of mechanical energy, the discovery of fire - the widespread use of chemical energy, and our present day state is characterized by use of atomic energy and an incomplete use of the entire energy available on our planet. A Dyson spherebuilding civilization and a conventional pan-galactic supercivilization (e.g., one that expands throughout its home galaxy by means of multiple Dyson spheres) are also shown. Presumably, the two last examples have not achieved the level of selfmodification that characterizes our Class 3 ETC; we describe such civilizations with the word "terraforming" to underline our wider interpretation, adjusting the environmental condition to one's needs in general, not necessarily on a planetary surface.

The bottom panel shows the Earth animals, with an offset from the pure Class 0 to account for tool usage, for example by chimpanzees. Present-day humanity spans the regions of using and modifying the environment, but stops short of the self-modifications. Barring major catastrophic events, we will probably reach that level of technology in the foreseeable future. Again, we mark the loci of Dyson sphere builders and terraforming pan-galactic supercivilization, and we add a hypothetical civilization that has converted its host galaxy into computational environments - the heat losses of such ETCs can potentially be detected by the WISE searches (e.g., Wright et al. 2014b, among others). The largest, all-encompassing class of civilizations is that of the adaptable, self-modifying ones. Changing the paradigm from physical change of environment to biological or even post-biological modification and optimization of the living organisms changes the energy requirements. Indeed, biological or computational research do not pose high energy demand. Our framework opens up a particularly interesting possibility: a selfmodifying civilization that does not need vast amounts of energy, because it is fully adaptable to the environment.

\section{Implications for our notion of ETCs}

The new classification proposed here is deeply rooted in humanity's own evolution and may be biased in ways that cannot be evaluated as long as we only know of one intelligent species: our own. Therefore, we assume that the lessons we learn from humanity's evolution and history are - at least to some degree - typical for at least some ETCs. The most obvious advantage of our scheme is the novel way of thinking about the ETC: we acknowledge that the parametric space that SETI searches need to cover cannot be described with a single parameter as Kardashev (1964) proposed. We introduce the question of how the available energy is used and what is its impact on the interaction with the matter in the Universe.

First, our classification scheme addresses the important question of detectability in a new way - the ultimate strength of Kardashev's work that was developed exactly to address this issue in the particular context of radio communications. Recently, Lingam \& Loeb (2019) concluded that the probability of detecting advanced ETCs' technosignatures may be two orders of magnitude lower than of detecting biosignatures from primitive life. It is worth remembering that all SETI projects have explored an exceptionally low fraction of the Milky Way parametric space that can be inhabited by ETCs - only $10^{-21}$ $10^{-18}$ (Wright et al. 2018). The realization that the footprint of an ETC and its detectability - both dominated mainly by the energy - may not scale up with the available energy, makes this estimate optimistic.

Another point, underlined by the new framework is that the two classifications - Kardashev's and the one proposed here are not directly correlated. In other words, the available amount of energy does not necessarily mean a more sophisticated interaction with matter and closer integration with the environment. The Kardashev classes are separated by vast 11-12 orders of magnitude, but humanity - still estimated to be using only about $70 \%$ of the energy at the disposal of a Class I civilization (Ćirković 2015) - does not seem too far from reaching the adequate biotechnological development to improve itself and to 
integrate with the environment. This is easy to understand if we keep in mind that biological research is not as energy intensive as nuclear physics, as pointed out above.

Furthermore, it is uncertain whether we can expect more available energy to only scale up our ability to modify the environment. In other words, we still lack the understanding of whether the building of a Dyson sphere is just a matter of having more powerful mining equipment and heavier rockets, or of some speculative technologies like nano-machines, selfreplicators, etc (see the discussion in Armstrong \& Sandberg 2013). In the former case, the total amount of available energy may play a role, but in the latter - less so, hereby removing any correlation between the two ETC scales described here.

These ideas are not entirely new among the SETI community. Indeed, the two-pointed arrow in Fig. 1 of Ćirković (2015) acknowledges the possibility that the complexity is not directly related to the available energy.

The third direct consequence from the broader consideration of ETCs' properties proposed here is the invalidation of the obvious statement that a Class III ETC does not exist in the Milky Way (Hart 1975). Our searches of such advanced ETCs rely on the concept of detecting their heat leaks (e.g., Wright et al. 2014a; Griffith et al. 2015), on the observational consequences from the controlled disintegration of galaxies for resources (Tipler 2003), or in searches for megastructures (Wright et al. 2016). The new classification scheme allows for the existence of quiet advanced civilizations that may co-exist with us, yet remain invisible to our radio, thermal, or transit searches. The implicit underlying assumption of Hart (1975) is that the hypothetical ETC is interacting with the matter on a similar level to us. We can not even speculate on whether it is possible to detect a heat leak or a transiting structure built by an ETC capable of interacting with the matter at sub-quark level. However, the answer is more likely to be negative, and not because that ETC would function according to some speculative physics laws, but because such an ETC would probably be vastly more efficient than us controlling its energy waste and minimizing its construction projects. Would such an advanced ETC even need megastructures and vast astroengineering projects?

It is also unlikely that the ongoing SETI project would successfully detect the Kardashev Class II stellivorous ETC described by Vidal (2016). Indeed, Heidmann \& Klein (1991) noted that a successful SETI search requires a match between the technology of the transmitting and receiving sides. These considerations cast some doubts on the popular pessimistic conclusions about the lack of ETCs (e.g., Lacki 2016; although some concerns for a Great Filter intrinsic to all civilizations appears to be still valid, e.g. Sotos 2019).

In summary, the new framework leads us to question the common assumption that progress is equivalent to ascending the ladder of energy consumption from Class I to III, as suggested by Dyson (1960) even before Kardashev came up with his classification. Indeed, an ETC can - as our own history shows it - progress from purely mechanical modification of its environment to more complex manipulation on chemical, atomic, nuclear, and other levels that allow it to achieve larger impacts, and more importantly, impacts that were not possible earlier with the simpler levels of interaction. However, this is not necessarily accomplished by ever increasing energy consumption - biosciences show this, and the opposite notion is probably a bias, due to the fact that astronomers and physicists akin to Kardashev, Dyson, and Sagan have been leading the SETI research, and they come armed with the idea that progress is embodied by more powerful accelerators or radio transmitters.
The final and the most important consequence of the new framework is the weakening of the Fermi Paradox (Hart 1975) if an ETCs' progress does not always imply higher energy consumption and waste, then progress does not imply higher detectability of the ETCs either. This explanation of the Fermi paradox opposes the usual conclusion for the rarity of ETCs. In fact, they may be common, but the low cross-section of our and their level of interaction with the Universe would account for the silentium universi.

\section{Predictions and implications for the SETI strategies}

The SETI programs search for ETCs' technosignatures - traces of advanced technologies. On the other hand, the searches for life at the crossing of the modern astronomical and biological research look for products from the natural life cycle (not necessarily advanced to the level of civilization, never mind how ill-defined this level may be; Des Marais et al. 2002; Seager et al. 2005; Segura et al. 2005; Scalo et al. 2007). However, the distinction between bio- and techno-signatures may not be clear-cut. Raup (1992) considered hypothetical animals that communicate with radio waves. Indeed, electric squid and rays (e.g., Raup 1992) use electricity, and direct electricity generation by biological systems has been demonstrated Tanaka et al. (2016). Therefore, signatures we commonly consider part of the technological realm, may actually evolve naturally. For simplification, we exclude this possibility from the following discussion, but we remind the reader that the most fundamental assumptions of SETI are not simple and straightforward.

What does this new ETC classification scheme mean for the definition of future SETI projects? Wittingly or not, the searches have so far been fine tuned to detect civilizations of Kardashev Types II and III, but only ones that follow the same "more-is-better" philosophy as we do. The mechanistic transfer of this power-hungry reasoning across a range of available energy levels wider than 25 orders of magnitude could be why these searches fail. The searches for Dyson spheres and swarms, although relatively easy with the present technology, also seem less than promising. A somewhat more productive strategy may be to search for biosignatures, because that would accompany life regardless of technological development (Lingam \& Loeb 2019). However, as discussed earlier, we cannot even fully rely on the biosignatures, because an advanced ETC can (presumably) easily modify itself to survive beyond the limitations of its original habitability limitations.

The possibilities of self-modification and for further integration of ETCs with their environment destroys the very idea of separating natural and artificial phenomena, and by definition makes it impossible for us to confidently detect any technosignatures, because (rephrasing A. C. Clarke) any advanced ETC will be indistinguishable from nature ${ }^{1}$. This idea of indistinguishability has been discussed earlier in Cirkovic $(2018)^{2}$. This process of technological development and optimization is different from the natural evolution of techno-like signatures proposed by Raup (1992).

\footnotetext{
1 The same rephrasing can be found in the 2011 essay by the Canadian writer and philosopher Karl Schroeder. Available at http://www . kschroeder . com/weblog/archive/2011/11/30/ the-deepening-paradox

2 The same idea can also be found in the 1971 essay The New Cosmogony by Polish writer and philosopher Stanislaw Lem. It is available in the collection A Perfect Vacuum (translation by M. Kandel), Northwestern U. Press, 1999, pp. 197-227.
} 
Undoubtedly, these arguments would be well understood by ETCs that have attained the levels of progress discussed here. Therefore, they would set up beacons to emit clearly artificial signals - on the condition (addressing this major question is beyond the scope of this work) that they still wish to communicate with less advanced counterparts such as us.

These arguments do not close the possibility of finding ETCs at a similar level to ours, but given the limited energy resources available to those ETC, such SETI programs are limited to a smaller space volume that can be searched with any hopes of success. SETI:

In conclusion, the new framework implies two strategies for

- Search for ETCs similar to us, for their radio radars and communications, for laser beacons and laser-powered interstellar probes, etc.

- Search for highly advanced ETCs (that have retained interest in their younger/simpler counterparts), for their energyefficient Benford beacons, rare/unstable element/isotope doped stars and white dwarfs, modulated/coordinated variables, etc.

\section{Summary and conclusions}

A classification for ETCs based on their level of interaction and integration with the environment is proposed. It can be combined with the classical Kardashev's scale to form a two-dimensional scheme for interpreting the ETC properties. The new framework makes it obvious that the available energy is not a unique measure of an ETC's progress, it may not even correlate with the quality of use of that energy. Furthermore, the possibility for progress without increased energy consumption implies a lower detectability, so in principle the existence of a Kardashev Type III ETC in the Milky Way cannot be excluded. This reasoning weakens the Fermi paradox, allowing for the existence of advanced, but energy-quiet ETCs.

The integration of ETCs with environment will make it impossible to tell apart the technosignatures from natural phenomena. Therefore, the only hope for future SETI searches to find advanced ETCs is to look for beacons, intentionally set up by them, to be found by backward civilizations like ours. It remains a matter of speculation if advanced ETCs would be interested in communicating with us. The other SETI window of opportunity is to search for ETCs at approximately our technological level.

This new proposal is not a criticism of Kardashev (1964). He carried out this work with the specific goal of estimating the feasibility of interstellar radio communications, and, naturally, it was used to evaluate the detectability of ETCs in radio. Undoubtedly, Kardashev's scale will continue to be important in the defininition of the sensitivities of SETI searches that utilize the strategies relying on communication leaks or communication beacons.

Acknowledgements. We thank the referees for the comments that helped to improve the paper and for pointing out the number of relevant works in the field of biology. We thank M.S. for the helpful discussions. D.M. acknowledges support from the BASAL Center for Astrophysics and Associated Technologies (CATA) through grant AFB 170002, and Proyecto FONDECYT No. 1170121 C.C. acknowledges support from DGI-UNAB project DI-11-19/R. JCB acknowledges support from project FONDECYT postdoctorado 3180716.

\section{References}

Adams, F. C., \& Laughlin, G. 1997, Rev. Mod. Phys., 69, 337

Annis, J. 1999, J. Br. Interplanet. Soc., 52, 33

Armstrong, S., \& Sandberg, A. 2013, Acta Astron., 89, 1

Barrow, J. D. 1999, Impossibility - The Limits of Science and the Science of Limits (Oxford: Oxford University Press)
Benford, J., Benford, G., \& Benford, D. 2010a, Astrobiology, 10, 475 Benford, G., Benford, J., \& Benford, D. 2010b, Astrobiology, 10, 491 Bradbury, R. J., Ćirković, M. M., \& Dvorsky, G. 2011, J. Br. Interplanet. Soc., 64, 156

Camps, C. C., Weisbach, N. R., Santinha, A. J., Danny, I., \& Platt, R. J. 2019 Nat. Methods, 16, 887

Carrigan, R. A., Jr. 2009, ApJ, 698, 2075

Ćirković, M. M. 2015, Serb. Astron. J., 191, 1

Cirkovic, M. M. 2018, The Great Silence: Science and Philosophy of Fermi's Paradox (Oxford: Oxford University Press )

Cocconi, G., \& Morrison, P. 1959, Nature, 184, 844

Davies, P. C. W., \& Wagner, R. V. 2013, Acta Astron., 89, 261

Des Marais, D. J., Harwit, M. O., Jucks, K. W., et al. 2002, Astrobiology, 2, 153

Dyson, F. J. 1960, Science, 131, 1667

Fabian, A. C. 1977, J. Br. Interplanet. Soc., 30, 112

Gaia Collaboration (Prusti, T., et al.) 2016, A\&A, 595, A1

Garrett, M. A. 2015, A\&A, 581, L5

Goodall, J. 1964, Nature, 201, 1264

Griffith, R. L., Wright, J. T., Maldonado, J., et al. 2015, ApJS, 217, 25

Hart, M. H. 1975, QJRAS, 16, 128

Heidmann, J., \& Klein, M. J. 1991, in Bioastronomy The Search for Extraterrestial Life - The Exploration Broadens (Berlin: Springer), 412

Hesketh, T., Lu, L., \& Xing, Z. W. 2011, Can. Med. Assoc. J., 183, 1374

Jinek, M., Chylinski, K., Fonfara, I., et al. 2012, Science, 337, 816

Jugaku, J., \& Nishimura, S. 2004, IAU Symp., 213, 437

Kahneman, D. 2011, Thinking, Fast and Slow, eds. Y. Terzian, \& E. Bilson (New York: FSG Adult), 499

Kardashev, N. S. 1964, Soviet Ast., 8, 217

Kecskes, C. 1998, J. Br. Interplanet. Soc., 51, 175

Kecskes, C. 2013, Asteroids, ed. V. Badescu (Berlin: Springer), 633

Kremers, H. M., Larson, D. R., Crowson, C. S., et al. 2015, J. Bone Jt, Surg., 97, 1386

Kunder, A., Kordopatis, G., Steinmetz, M., et al. 2017, AJ, 153, 75

Kwon, J. Y., \& Bercovici, H. L. 2017, Front. Psychol., 8, 2308

Lacki, B. C. 2016, ArXiv e-prints [arXiv:1604 . 07844]

Lacki, B. C. 2019, PASP, 131, 024102

Lingam, M., \& Loeb, A. 2019, Astrobiology, 19, 28

McGrew, W. C. 1974, J. Hum. Evol., 3, 501

Morgana, I. G., Frenchc, A. N., Ashby, R. S., et al. 2018, Prog. Retin. Eye Res., 62,134

Neal, M. 2014, Risk Manag., 16, 63

Nunn, A. V. W., Guy, G. W., \& Bell, J. D. 2014, Nutr. Metab., 11, 34

Olson, S. J. 2017, Int. J. Astrobiol., 16, 176

O’Neill, G. K., \& Kraus, J. 1979, Cosmic Search, 1, 16

Osmanov, Z. 2016, Int. J. Astrobiol., 15, 127

Osmanov, Z., \& Berezhiani, V. I. 2018, Int. J. Astrobiol., 17, 356

Planck Collaboration XIII. 2016, A\&A, 594, A13

Raup, D. M. 1992, Acta Astronaut., 26, 257

Sagan, C., \& Walker, R. G. 1966, ApJ, 144, 1216

Sandberg, A., Armstrong, S., \& Cirkovic, M. M. 2017, J. Br. Interplanet. Soc., submitted [arXiv:1705.03394]

Scalo, J., Kaltenegger, L., Segura, A. G., et al. 2007, Astrobiology, 7, 85

Seager, S., Turner, E. L., Schafer, J., \& Ford, E. B. 2005, Astrobiology, 5, 372

Segura, A., Kasting, J. F., Meadows, V., et al. 2005, Astrobiology, 5, 706

Slysh, V. I. 1985, IAU Symp., 112, 315

Sotos, J. G. 2019, Int. J. Astrobiol., 18, 445

Strecker, J., Ladha, A., Gardner, Z., et al. 2019, Science, 365, 48

Suffern, K. G. 1977, PASA, 3, 177

Tanaka, Y., Funano, S., Nishizawa, Y., et al. 2016, Sci. Rep., 6, 25899

Tilgner, C. N., \& Heinrichsen, I. 1998, Acta Astron., 42, 607

Timofeev, M. Y., Kardashev, N. S., \& Promyslov, V. G. 2000, Acta Astron., 46, 655

Tipler, F. J. 2003, Int. J. Astrobiol., 2, 141

Van Lawick-Goodall, J. 1971, Adv. Study Behav., 3, 195

Vidal, C. 2016, Acta Astron., 128, 251

Wengler, A., Nimptsch, U., \& Mansky, T. 2014, Dtsch. Ärztebl. Int., 111, 407

Williams, K. M., Bertelsen, G., Cumberland, P., et al. 2015, Ophthalmology, 122, 1489

Wright, E. L., Eisenhardt, P. R. M., Mainzer, A. K., et al. 2010, AJ, 140, 1868

Wright, J. T., Mullan, B., Sigurdsson, S., \& Povich, M. S. 2014a, ApJ, 792, 26

Wright, J. T., Griffith, R. L., Sigurdsson, S., Povich, M. S., \& Mullan, B. 2014b, ApJ, 792, 27

Wright, J. T., Cartier, K. M. S., Zhao, M., Jontof-Hutter, D., \& Ford, E. B. 2016, ApJ, 816, 17

Wright, J. T., Kanodia, S., \& Lubar, E. 2018, AJ, 156, 260

Zackrisson, E., Calissendorff, P., Asadi, S., \& Nyholm, A. 2015, ApJ, 810, 23

Zackrisson, E., Korn, A. J., Wehrhahn, A., \& Reiter, J. 2018, ApJ, 862, 21 